\title{
Development of Subclinical Hyperthyroidism due to Graves' Disease in a Hypothyroid Woman who had Undergone Hemithyroidectomy for Adenomatous Goiter and Radiotherapy for Nasopharyngeal Cancer
}

\author{
YOSHINORI NAKAGAWA, KOUKI MORI, SAEKO HOSHIKAWA, HIROSHI OZAKI, SADAYOSHI ITO \\ AND KATSUMI YOSHIDA* \\ Division of Nephrology, Endocrinology and Vascular Medicine, Tohoku University Graduate School of Medicine, Sendai 980-8574, \\ Japan \\ *Department of Medical Technology, Tohoku University School of Health Sciences, Sendai 980-8575, Japan
}

\begin{abstract}
A variety of thyroid disorders develop following external radiation to head and neck cancers. Hypothyroidism is the most common clinical consequence of the radiotherapy and lifelong thyroid hormone replacement is required in many cases. Patients who received both hemithyroidectomy and the external radiation to the neck are at especially high risk for permanent hypothyroidism. Here we report an unusual case with radiation-induced hypothyroidism who had undergone hemithyroidectomy for adenomatous goiter 8 years before the radiotherapy for nasopharyngeal cancer and subclinical hyperthyroidism due to Graves' disease developed during thyroxine replacement therapy. Thus subclinical hyperthyroidism due to Graves' disease can develop in patients with radiation-induced hypothyroidism even if they have undergone hemithyroidectomy for thyroid nodules. Therefore careful and periodic evaluation of thyroid function is required even on adequate thyroxine replacement therapy.
\end{abstract}

Key words: Radiotherapy, Hypothyroidism, Graves’ disease, Thyroidectomy

(Endocrine Journal 54: 35-37, 2007)

IT is well known that a variety of thyroid disorders may develop following external radiation to head and neck cancers [1, 2]. Hypothyroidism is the most common clinical consequence of the radiotherapy; the incidence of radiation-induced hypothyroidism varies widely, but most frequently is in the range of 20 to $30 \%$ $[1,2]$. Thus in many patients with radiation-induced hypothyroidism, lifelong thyroid hormone replacement is required. Hemithyroidectomy is especially associated with an increased risk to develop postradiotherapy hypothyroidism $[3,4]$. On the other hand, Graves' hy-

Received: July 18, 2006

Accepted: September 7, 2006

Correspondence to: Kouki MORI, M.D., Ph.D., Division of Nephrology, Endocrinology and Vascular Medicine, Tohoku University Graduate School of Medicine, 1-1 Seiryo-machi, Aoba-ku, Sendai 980-8574, Japan perthyroidism can develop after external radiation to the neck $[1,2]$. Here we report the development of subclinical hyperthyroidism due to Graves' disease in a woman on thyroxine replacement for radiation-induced hypothyroidism, who had undergone hemithyroidectomy for adenomatous goiter 8 years before the radiotherapy for nasopharyngeal cancer.

\section{Case Report}

At the age of 33, a Japanese woman underwent a right hemithyroidectomy for adenomatous goiter in 1989. At the beginning of 1997, she noticed a large tumor in the right neck. Extensive evaluation revealed nasopharyngeal cancer in stage IV. She received external radiation therapy to the neck including the thyroid region (total $70 \mathrm{~Gy}$ ) combined with chemotherapy us- 


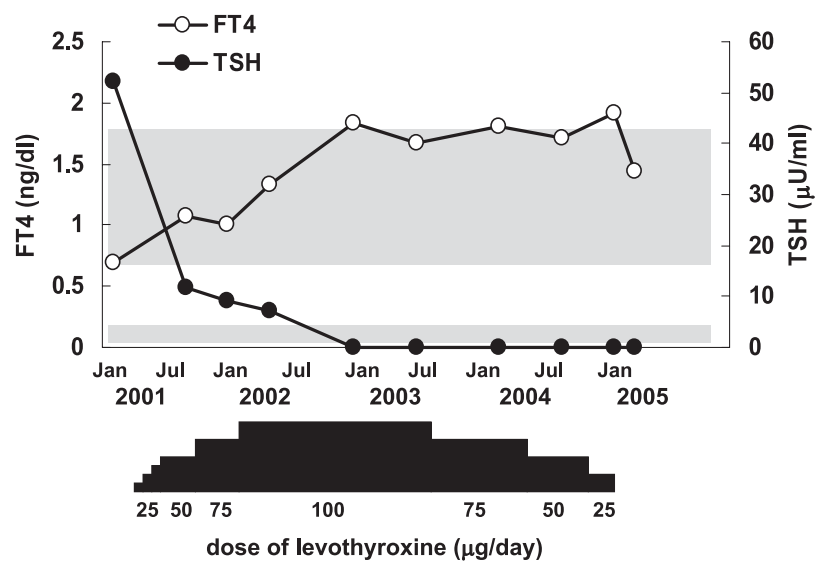

Fig. 1. Serum free T4 (FT4) and TSH concentrations and replacement therapy with levothyroxine. The shaded areas represent the normal ranges of serum FT4 (0.7-1.8 $\mathrm{ng} / \mathrm{dl}$; upper) and serum TSH $(0.3-3.5 \mu \mathrm{U} / \mathrm{ml}$; lower $)$.

Table 1. Retrospective studies showing the presence of TSH receptor antibodies

\begin{tabular}{lcccc}
\hline & & TBII (IU/l) & TSAb (\%) & TSBAb (\%) \\
\hline 2001 & Aug & 3 & 180.1 & 14.9 \\
2002 & Apr & 5.4 & 150.2 & 35.3 \\
& Dec & 24 & 376.5 & 37.9 \\
\multirow{2}{*}{ Reference range } & $<1.0$ & $<180$ & $<40$ \\
\hline
\end{tabular}

Abbreviations: TBII, TSH binding inhibitory immunoglobulin; $\mathrm{TSAb}$, thyroid stimulating antibody; TSBAb, thyroid stimulation blocking antibody.

ing cisplatin. The therapy was very effective, and the tumor disappeared completely. In February 2001, she was aware of facial edema, lassitude, cold intolerance and unexplained weight gain. Laboratory tests revealed slightly decreased serum free T4 (FT4) and increased serum TSH levels (Fig. 1) at which time she was referred to our clinic for the treatment of hypothyroidism. Thyroid was not palpable. Antithyroglobulin $(\mathrm{TgAb})$ and anti-thyroid peroxidase (TPOAb) antibodies were negative (data not shown). Thus a diagnosis of radiation-induced hypothyroidism was made and replacement therapy with levothyroxine (L-T4) was initiated (Fig. 1). In December 2002, her serum TSH level was markedly decreased, and the dose of L-T4 was gradually decreased thereafter. As shown in Fig. 1, however, her TSH levels were still suppressed even after the termination of the replace- ment therapy. Additional studies revealed the presence of TSH binding inhibitory immunoglobulin (TBII; $856 \mathrm{IU} / \mathrm{L}$ ) and an increased uptake of radioactive technetium in the residual left lobe of the thyroid gland. Thus a diagnosis of Graves' disease was made. Orbitopathy was absent. Retrospective examination using stored sera confirmed that TBII and thyroid stimulating antibody (TSAb) had been present since 2001 (Table 1). Thyroid stimulation blocking antibody (TSBAb) was absent. She does not receive antithyroid therapy because of normal serum FT4 levels and by her own choice.

\section{Discussion}

Here we report a patient with radiation-induced hypothyroidism followed by subclinical hyperthyroidism due to Graves' disease. While spontaneous recovery from hypothyroidism is common in patients with Hashimoto's thyroiditis [5], such recovery is not seen in patients with radiation-induced hypothyroidism. Our patient was especially at a high risk for permanent hypothyroidism since she had received both hemithyroidectomy and external radiation to the neck $[3,4]$. In our case, it is quite possible that hypothyroidism was caused by irradiation, but not by thyroid autoimmunity, since TSBAb, TgAb and TPOAb were absent in her sera. Gradual increase in the levels of TBII may result in the stimulation of residual, but still functional thyroid follicles, allowing them to produce thyroid hormone to the level regarded as subclinical hyperthyroidism. Previous studies have demonstrated that Graves' hyperthyroidism can occur in hypothyroid patients in whom external irradiation to the neck was performed for the treatment of Hodgkin's disease [6, 7]. However, they do not show whether hyperthyroidism developed in hypothyroid patients who had undergone hemithyroidectomy prior to irradiation to the neck.

In conclusion, subclinical hyperthyroidism due to Graves' disease can develop in hypothyroid patients in whom both hemithyroidectomy and radiotherapy for head and neck cancers have been performed. Accordingly, careful and periodic evaluation of thyroid function is required in such patients, even on adequate thyroxine replacement therapy. 


\section{References}

1. Hancock SL, McDougall IR, Constine LS (1995) Thyroid abnormalities after therapeutic external radiation. Int J Radiation Oncol Biol Phys 31: 1165-1170.

2. Jereczek-Fossa BA, Alterio D, Jassem J, Gibelli B, Tradati N, Orecchia R (2004) Radiotherapy-induced thyroid disorders. Cancer Treat Rev 30: 369-384.

3. Weissler MC, Berry BW (1991) Thyroid-stimulating hormone levels after radiotherapy and combined therapy for head and neck cancer. Head Neck 13: 420-423.

4. Turner SL, Tiver KW, Boyages SC (1995) Thyroid dysfunction following radiotherapy for head and neck cancer. Int J Radiation Oncol Biol Phys 31: 279-283.
5. Yoshinari M, Okamura K, Tokuyama T, Shiroozu A, Nakashima T, Inoue K, Omae T (1983) Clinical importance of reversibility in primary goitrous hypothyroidism. Br Med J 287: 720-722.

6. Hancock SL, Cox RS, McDougall IR (1991) Thyroid diseases after treatment of Hodgkin's disease. $N$ Engl J Med 325: 599-605.

7. Constine LS, McDougall IR (1982) Radiation therapy for Hodgkin's disease followed by hypothyroidism and then Graves' hyperthyroidism. Clin Nucl Med 7: 6970 . 